TABLE 1 Inhibitor sensitivity of ${ }^{14} \mathrm{C}$-TEA uptake in Xenopus oocytes injected with CRNA of the renal organic cation transporter OCT1

Inhibitor

$K_{\mathrm{i}}(\mu \mathrm{M})$

Cyanine-863

Decynium-22

Tetrapentylammonium

Quinine

Desipramine

Mepiperphenidol

Procainamide

1-Methyl-4-phenylpyridinium

Corticosteron

Reserpine

O-methyl-isoprenaline

Tetramethylammonium

$N^{1}$-methylnicotinamide

Xenopus oocytes were injected with $5 \mathrm{ng}$ OCT1 cRNA and the effect of 5-8 different inhibitor concentrations on the uptake of $95 \mu \mathrm{M}{ }^{14} \mathrm{C}$ TEA into the oocytes was measured as for Fig. 1f. Inhibition curves were fitted by nonlinear regression analysis and the $K_{\mathrm{i}}$ values ( \pm s.e.m.) calculated.

between TM3 and TM4 has been found in an OCT1-homologous cDNA from human kidney (our unpublished results) which may also exist in OCT1 (Fig. 2c). With this transmembrane domain included, there are several possible intracellular protein kinase $\mathrm{C}$ phosphorylation sites which may be involved in transport regulation ${ }^{19}$. Also, three short sequence motifs in OCT1 that have been identified in the cytoplasmic domains of transport proteins from different families ${ }^{20}$ become localized intracellularly. Northern blot analysis (Fig. $3 a-d$ ) showed bands of 1.9, 3.4 and 4.8 kilobases $(\mathrm{kb})$ in samples of kidney cortex, kidney medulla, liver, intestine and colon of rat. Porcine LLC-PK 1 cells gave a single hybridization band at $3.4 \mathrm{~kb}$. Extra-neuronal noradrenaline transport in heart and Caki-1 cells has been described that has the functional properties of renal luminal $\mathrm{H}^{+}$-cation antiport ${ }^{2123}$, but as we detected no hybridization in heart and Caki-1 cells, OCT1 probably belongs to a different genetic family. We amplified a cDNA fragment from rat liver by polymerase chain reaction which was identical to nucleotides 400620 of OCT1, indicating that OCT1 is probably also expressed in liver. The uptake of organic cations in primary cultured rat liver hepatocytes has been found to be almost exclusively performed by a system with $K_{\mathrm{i}}$ values nearly identical to OCT 1 for the inhibitors $O$-methyl-isoprenaline, MPP, quinine, decynium-22 and cyanine-863 (E. Martel, H. Russ, I. Azevedo and E. Schömig, manuscript in preparation). This OCT1 fingerprint of inhibitory constants indicates that OCT1 could be the main transporter responsible for organic cation uptake into hepatocytes. In situ hybridization in rat kidney showed that OCT1 is expressed in proximal tubules but apparently not in distal tubules, collecting ducts and glomeruli (Fig. 3e,f). OCT1 is expressed in hepatocytes in liver (Fig. $3 g, h$ ) and in enterocytes of villi and crypts in small intestine (Fig. 3i,j).

Our results indicate that OCT1 is a new type of polyspecific transporter which is involved in the elimination of cationic drugs. It shares common features with organic cation uptake over the basolateral membrane of renal proximal tubules and appears to be identical to the main organic cation uptake system in hepatocytes. Expression of the human OCT1-homologous gene and of the renal luminal organic cation transporter in epithelial cell lines will provide in vitro test systems for the development of drugs with optimized excretion and minimized nephrotoxicity.

\footnotetext{
Received 29 April; accepted 18 October 1994

1. Roch Ramel, F., Besseghir. K. \& Murer, H. in Handbook of Physiology 8, Renai Physiology vol. 2 (ed. Windhager, E. E.) 2189-2262 (Oxford Univ. Press, Oxford 1992) 2. Ullrich, K. J. Biochim. biophys. Acta 1197, 45-62 (1994).
}

3. Pritchard, J. B. \& Miller, D. S. in The Kidney: Physiology and Pathophysiology (eds Seldin. D. W. \& Giebisch, G.) 2921-2945 (Raven, New York, 1992).

4. Meijer, D. K. F., Mol, W. E. M., Müller, M. \& Kurz, G. J. Pharmakin. Biopharmac. 18, 35 70 (1990).

5. Schuldiner, S. J. Neurochem. 62, 2067-2078 (1994

6. Schloss, P., Mayser, W. \& Betz, H. FEBS Lett. 307, 76-80 (1992)

7. Gottesman, M. M. \& Pastan, I. A. Rev. Biochem. 62, 385-427 (1993)

8. Wright, S. H. \& Wunz, T. M. Am. J. Physiol. 253, F1040-F1050 (1987)

9. Montrose-Rafizaden, C., Mingard, F., Murer, H. \& Roch-Ramel, F. Am. J. Physiol. 257, F243F251 (1989).

10. Takano, M., Inui, K.-I., Okano, T., Saito. H. \& Hori, R. Biochim. biophys. Acta 773, 113 124 (1984)

1. Sokol, P. P. \& McKinney, T. D. Am. J. Physiol. 258, F1599-F1607 (1990).

12. Thiebaut, F. et al. J. Histochem. Cytochem. 37, 159-164 (1989).

13. Ullrich, K. J., Papavassiliou, F., David, C., Rumrich, G. \& Fritzsch, G. Pfluegers Arch. 419, 84-92 (1991)

14. David. D., Rumrich, G. \& Ullrich, K. J. Krongreß3 der Gesellschaft für Nephrologie Hamburg. Abstr. 472 (Dustri, München, 1994)

15. Pacholczyk, T. Blakely, R. D. \& Amara, S. G. Nature 350, 350-354 (1991)

16. Schömig, E., Babin-Ebell, J. \& Russ, H. Naunyn-Schmiedeberg's Arch. Pharmac. 347, 379 383 (1993).

17. Magagnin, S. et al. J. biol. Chem. 267, 15384-15390 (1992).

18. Kozak, M. Nucleic Acids Res, 12, 857-872 (1984).

19. Hohage, H., Mörth, D. M., Querl. I. U. \& Greven, J. Pharmac. Exp. Ther. 268, 897-901 (1994)

20. Gingrich, J. A. et al. FEBS Lett. 312, 115-122 (1992)

21. Schömig, E. \& Schönfeld, C.-L. Naunyn-Schmiedeberg's Arch. Pharmac. 341, 404-410 (1990)

22. Trendelenburg, U. in Handbook of Experimental Pharmacology (eds Trendelenburg, U. \& Weiner, N.) 279-319 (Springer. Berlin, 1988).

23. Russ, H., Gliese, M., Sonna, J. \& Schömig, E. Naunyn-Schmiedeberg's Arch. Pharmac. 346, $158-165$ (1992).

24. Veyhl, M. et al. J. biol. Chem. 268, 25041-25053 (1993).

25. Kyte, J. \& Doolittle, R. F. J. molec. Biol. 157, 105-132 (1982)

26. Sanger, F., Nicklen, S. \& Coulson, A. R. Proc. natn. Acad. Sci. U.S.A. 74, 5463-5467 (1977).

27. Chomczynski, P. \& Sacchi, N. Anaiyt. Biochem. 162, 156-159 (1987),

ACKNOWLEDGEMENTS. We thank K. J. Ullich, who initiated this study when the authors were working at the Max-Planck-Institut für Biophysik in Frankfurt, for his support, and W. Schwarz ce reported in this letter has been submitted to the GenBank/EMBL Data Bank under the accession number X78855.

\section{Systemic and mucosal immunity induced by BCG vector expressing outer-surface protein A of Borrelia burgdorferi}

\section{Solomon Langermann*, Susan Palaszynski*, Ariadna Sadziene $\dagger$, C. Kendall Stover* ${ }^{*}$ \& Scott Koenig*}

* Medimmune Inc., Gaithersburg, Maryland 20878, USA

$\dagger$ Department of Microbiology, The University of Texas Health Science Center at San Antonio, San Antonio, Texas 78284, USA

THE bacillus Calmette-Guerin (BCG) is a live attenuated strain of Mycohacterium bovis which offers potential advantages as a vector for mucosal delivery of antigens ${ }^{1-3}$. Recombinant BCG elicits protective humoral immune responses to a variety of antigens ${ }^{4}$. Furthermore, BCG binds specifically to microfold cells ${ }^{5}$ present in the epithelium overlying lymphoid follicles throughout the mucosal immune system ${ }^{6-8}$. Here we show that a single intranasal vaccination with recombinant BCG expressing the outersurface protein $A$ antigen from $B$. burgdorfer $i^{9}$ results in a prolonged (more than one year) protective systemic IgG response and a highly sustained secretory $\operatorname{IgA}$ response which is disseminated throughout the mucosal immune system. Furthermore, intranasal immunization induces marked, organized lymphocyte accumulation in the proximal nasopharyngeal lymphoid tissue as well as at distal mucosal sites; the appearance and persistence of lymphoid aggregates correlates with the secretory immune responses. Thus 
intranasal immunization with recombinant BCG is a powerful method for inducing long-lasting secretory and systemic immune responses.

We previously demonstrated that parenteral immunization of mice with an optimal dose of $2 \times 10^{6} \mathrm{CFU}$ recombinant $\mathrm{BCG}$ expressing outer-surface protein A ( $\mathrm{rBCG}-\mathrm{OspA}$ ) resulted in that monoclonal and polyclonal sera specific for OspA protect mice against challenge with $B$. burgdorferi $i^{11,12}$. Intranasal (i.n.) delivery of $2 \times 10^{8} \mathrm{CFU}$ yields similar systemic IgG responses, both in magnitude and kinetics, to those seen when $2 \times 10^{6} \mathrm{CFU}$ are inoculated intraperitoneally (i.p.) (Fig. 1a). A $10^{6} \mathrm{CFU}$ dose of $\mathrm{rBCG}-\mathrm{OspA}$ delivered i.n. elicited a comparable immune response to the $10^{8} \mathrm{CFU}$ dose over time; however, the time-topeak response was delayed by $4-6$ weeks (Fig. 1b). The antiOspA IgG antibodies stimulated by i.n. immunization exhibited strong in vitro growth-inhibiting titres against $B$. burgdorferi strain B31 (ref. 13) and i.n.-immunized mice were completely protected from infection by the infectious Sh.2 strain of $B$. burgdorferi ${ }^{14}$ (Table 1). Intranasal immunization also resulted in a highly sustained, low-titre serum $\operatorname{IgA}$ response against OspA (Fig. 1a). Intraperitoneal immunization with $10^{6} \mathrm{CFU}$ rBCG-OspA did not result in any measurable serum IgA titre to OspA (Fig. 1). Furthermore, i.n. immunization induced high levels of OspA and BCG-specific IgA spot-forming cells (SFC) in the lungs (Fig. 2), indicative of a strong secretory IgA response ${ }^{15-18}$. The secretory IgA response could be detected as early as 6 weeks and as late as 22 weeks (the latest time tested) after immunization. Intraperitoneal immunization did not yield high levels of protective anti-OspA IgG antibodies ${ }^{10}$. These findings were consistent with earlier passive transfer studies showing

any OspA or BCG-specific IgA SFC, even at a dose of $10^{8} \mathrm{CFU}$.

Mononuclear cells isolated from the lamina propria of the gastrointestinal tract of i.n.-immunized mice contained high frequencies of OspA-specific IgA SFC as measured by an ELISPOT assay. Between $10^{2}$ and $10^{3}$ OspA-specific SFC per $10^{6}$ cells were detected at 9 and 16 weeks after immunization (data not shown). No OspA-specific IgA plasma cells were detected in the gut of i.p.-immunized mice. IgA against OspA was also found in vaginal washes of i.n.-immunized, but not i.p.immunized mice, as early as 6 weeks post-immunization. A total of $0.08 \mu \mathrm{g} \mathrm{ml}^{-1}$ of anti-OspA IgA was detected in pooled, vaginal secretions ${ }^{19}$ from three i.n.-immunized mice which represented $\sim 8 \%$ of the total amount of $\operatorname{IgA}$ detectable in the vaginal tract. Antigen-specific vaginal IgA responses persisted for at least 19 weeks.

Unlike i.n. immunization, oral delivery of $\mathrm{rBCG}\left(10^{7} \mathrm{CFU}\right)$ in bicarbonate buffer elicited low levels of OspA-specific systemic $\operatorname{IgG}$ (end-point titres 1/400); low levels of antigen-specific IgA were found in the gastrointestinal tract, as measured by assaying for antigen-specific IgA in fecal-pellet extracts ${ }^{19}$ (data not shown), but not at other mucosal sites (Fig. 2). These low titres may be due to decreased survival of $\mathrm{BCG}$ in the gastrointestinal tract; either a tenfold increase in the dose of BCG $\left(10^{8} \mathrm{CFU}\right)$ or pretreatment of mice with the histamine receptor antagonist cimetidine (followed by $10^{7} \mathrm{CFU}$ BCG) resulted in a fourfold enhancement in systemic IgG responses $(1: 6,400)$.

Microscopic and gross anatomical analysis of lung tissue from i.n.-immunized mice revealed discrete foci of lymphocytic infiltrates (Fig. 3a). These lymphoid aggregates were highly organized in a focal perivascular and peribronchiolar distribution and

FIG. 1 Serum IgG and IgA titres to OspA following intranasal (i.n.) or intraperitoneal (i.p.) immunization with rBCG-OspA or with non-rBCG. a, Persistence of serum IgG levels against OspA in BALB/c mice immunized either i.n. or i.p. with the $\mathrm{rBCG}-\mathrm{OspA}$ vaccine versus persistence of the serum IgA response only in the i.n.-immunized mice. $b$, Dose response for mice immunized intranasally with rBCG-0spA2619 (serum IgG levels). The serum immune responses to OspA were determined for pooled serum samples from five individual mice in each group, every two to four weeks post-immunization, by enzyme-linked immunosorbant assays (ELISAs). Antibody titres shown in a were determined up to week 52 (for i.p.-immunized) or week 63 (i.n.-immunized), the latest time points evaluated.

METHODS. ELISA plates (Immulon 1) were coated with $50 \mu \mathrm{l}$ OspA protein $\left(0.1 \mu \mathrm{g} \mathrm{ml}^{-1}\right.$; Connaught, Swiftwater, PA) or $50 \mu \mathrm{l}$ of a $1 / 500$ dilution of whole BCG lysate $\left(10^{9} \mathrm{CFU}\right)$ suspended in $0.1 \mathrm{M}$ carbonate buffer $\left(\mathrm{pH}\right.$ 9.6) and incubated overnight at $4{ }^{\circ} \mathrm{C}$. The antigen solution was removed and plates were incubated with blocking solution $(0.5 \% \mathrm{BSA}$ and $0.5 \%$ non-fat dry milk $+0.2 \%$ sodium azide in $\mathrm{H}_{2} \mathrm{O}$ ) for $1 \mathrm{~h}$ at room temperature. Twofold serial dilutions starting at either 1:10 (IgA assays) or 1:200 (IgG assays) of pooled serum from five individual BALB/c mice in each group, were prepared in blocking solution and $50 \mu \mathrm{l}$ of each dilution was added to duplicate wells of the antigen-coated plate and incubated at room temperature for $1 \mathrm{~h}$; for all groups in $a$ and $b$, the standard error of the mean titre for individual mice within a group did not exceed $4.3 \%$. Plates were then washed with PBS $+0.1 \%$ Tween-20 (PBS-T20) and incubated with $50 \mu \mathrm{l}$ of a 1:4,000 dilution of peroxidaseconjugated goat anti-mouse IgG or IgA (Southern Biotechnology Associates) for $1 \mathrm{~h}$. Plates were developed with 2, 2'-azino-di[3-ethyl-benzthiazoline sulphonate (6)] (ABTS) substrate reagent (Kierkegaard and Perry Labs) and absorbance was measured at $405 \mathrm{~nm}\left(A_{405}\right)$ on a Dynatech ELISA reader. Endpoints were defined as the highest dilution at which the $A_{405}$ values were twice the values for preimmune sera diluted 1:10 or 1:200 in blocking solution. Serum from mice immunized with nonrBCG did not react with the OspA antigen, as shown. Intranasally immunized mice were inoculated with a dose of $1 \times 10^{8}$ CFU rBCGOspA2619S or non-recombinent BCG in $50 \mu \mathrm{l}$ PBS on 2 consecutive days (total dose, $2 \times 10^{8} \mathrm{CFU}$ ). Intraperitoneally immunized mice received a total of $2 \times 10^{6} \mathrm{CFU}$ in $100 \mu \mathrm{l}$ PBS. In the rBCG vaccine strain (rBCG-2619SOspA) used in these experiments, OspA is expressed as a surface-associated lipoprotein similar to the rBCG-p19PSOspA described earlier ${ }^{10}$, except that OspA expression in rBCG-2619SOspA is driven by the mycobacterial $h s p 60$ promoter. Only BALB/c mice were
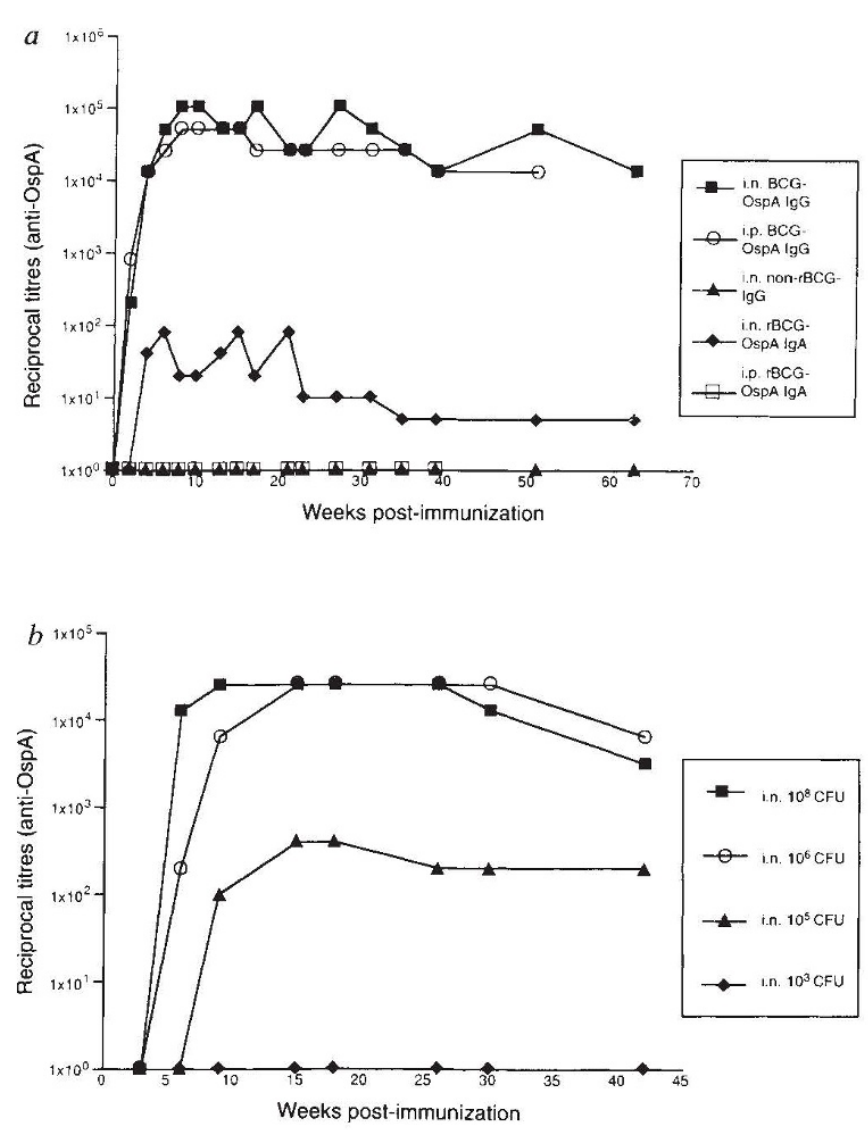

included in these immunogenicity studies on the basis of a previous survey of immune responsiveness to OspA among 24 inbred and outbred mouse strains ${ }^{10}$. 
TABLE 1 In vitro growth inhibition titres and protection from intradermal challenge with $B$. burgdorferi

\begin{tabular}{cccccc}
\hline rBCG vector/route & $\begin{array}{c}\text { Growth } \\
\text { of immunization }\end{array}$ & $\begin{array}{c}\text { Plasma } \\
\text { inhibition titre }\end{array}$ & $\begin{array}{c}\text { Heart } \\
\text { culture }(+) / \text { total }\end{array}$ & $\begin{array}{c}\text { Bladder } \\
\text { culture }(+) / \text { total }\end{array}$ & $\begin{array}{c}\text { Tibiotarsal joint } \\
\text { culture }(+) / \text { total }\end{array}$ \\
rBCG-OspA/i.n. & $1: 16,384$ & $0 / 7$ & $0 / 7$ & $0 / 7$ & $0 / 7$ \\
rBCG-OspA/i.p. & $1: 32,768$ & $0 / 7$ & $0 / 7$ & $0 / 7$ & $0 / 7$ \\
Non-rBCG/i.n. & $<1: 8$ & $3 / 7$ & $3 / 7$ & $7 / 7$ & $6 / 7$ \\
Non-immunized & $1: 64$ & $1 / 7$ & $3 / 7$ & $7 / 7$ & $6 / 7$ \\
\hline
\end{tabular}

In vitro growth inhibition titres against the $B 31$ strain of $B$. burgdorferi were determined as described ${ }^{11}$ using pooled sera from mice immunized i.n. or i.p. with rBCG-OspA, mice immunized i.n. with non-rBCG, or non-immunized mice. 96 -well, flat-bottom plates were seeded with $2 \times 10^{6}$ spirochetes in $0.1 \mathrm{ml}$ BSK-II medium derived from late log-phase culture of a high-passage isolate of B. burgdorferi B31 (ATCC35210) grown in the same medium. Twofold serial dilutions were added to duplicate wells, and the plates incubated at $34{ }^{\circ} \mathrm{C}$ for $72 \mathrm{~h}$ in the presence of $1 \% \mathrm{CO}_{2}$. Bacterial growth was monitored by colour changes in phenol red indicator dye. Growth inhibition titres shown are complement-dependent; complement-independent titres for mice immunized i.n. or i.p. with rBCG-OspA were 1:3,200 and 1:6,400 respectively. For challenge experiments, immunized and age-matched, non-immunized control BALB/c mice were challenged intradermally (i.d.) at the base of the tail, 13 weeks after immunization, with $10^{4}$ spirochetes $\left(\sim 100 / D_{50}\right.$ units) derived from the low-passage Sh. 2 strain of $B$. burgdorferi ${ }^{12}$; there were 7 mice per group. Mice were killed 14 days after challenge and their hearts, bladders and tibiotarsal joint tissues were minced and cultured together with blood samples from each animal in BSK-II medium. Cultures were monitored up to day 14 by phase-contrast microscopy for the presence of spirochetes. The presence of even a single spirochete in any of twenty fields was scored as a positive infection. Challenge experiments done as late as 25 and 40 weeks post-immunization also demonstrated sterile immunity in rBCG-OspA-immunized mice (G. Bansal, Medlmmune). Data are presented as number of mice giving positive cultures at a particular site per total mice in the group; some of the mice in the non-immunized and control nonrBCG groups had positive cultures at more than one site, and all control mice had positive bladder cultures.

contained a mixture of monocytes, macrophages, activated lymphocytes and plasma cells (Fig. $3 b, c$ ). The appearance of lymphoid aggregates correlated with the emergence of systemic and local immune responses to $\mathrm{rBCG}$. The lymphoid aggregates persisted at least 22 weeks after immunization. Despite vigorous recruitment of immune cells into the lungs of i.n.-immunized mice, there was no evidence of fibrosis or granuloma formation over a 22-week period.

Flow cytometric analysis (fluorescence-activated cell sorting, FACS) of $10^{6}$ intraparenchymal lung lymphocytes from i.n.immunized, i.p.-immunized or naive mice revealed a threefold increase of B cells in the lungs of mice immunized i.n. with either $\mathrm{rBCG}$ or BCG lacking the recombinant vector, compared to lungs isolated from i.p.-immunized mice or naive mice (data not shown). Periodic acid-Schiff base staining showed a significant number of plasma cells, consistent with the FACS analysis. FACS revealed no significant increases in the percentage of $\mathrm{CD}^{+}$and $\mathrm{CD}^{+}$cells in the lungs of i.n.-immunized mice relative to naive or i.p.-immunized mice $(\sim 25$ and $5-10 \%$, respectively, in all cases). No differences were noted in the CD4/CD8 ratios among the different groups.

FIG. 2 Secretory immune response to OspA occurs in respiratory tract only when mice are immunized i.n. as determined by an ELISPOT assay. Results are expressed as the mean number \pm s.e.m. of spot-forming cells (SFC/IgA-producing) per $10^{6}$ cells (intraparenchymal lung lymphocytes (IPLLS)), the limit of detection of the assay. i.n., intranasal; i.g., intragastric; i.p., intraperitoneal.

METHODS. The ELISPOT assay was used to determine the number of cells spontaneously secreting antigen-specific IgA tissues of mucosally immunized mice (secretory immune response) ${ }^{23,24}$. 96-well flat-bottom plates with a nitrocellulose base (Millititer-HA, Millipore) were coated overnight at $4{ }^{\circ} \mathrm{C}$ with either OspA protein $\left(0.1 \mu \mathrm{g} \mathrm{ml} l^{-1}\right)$ or a $1 / 500$ dilution of whole BCG lysate in PBS $(100 \mu \mathrm{l}$ per well). Plates were washed with PBS $(\times 3)$ and incubated for $1 \mathrm{~h}$ with blocking solution (RPMI $1640+10 \%$ fetal calf serum). After removal of the blocking solution, lymphocytes isolated from lung tissue of a total of 3 mice per group were pooled and added at varying concentrations ranging from $1 \times 10^{2}-1 \times 10^{5}$ lymphocytes per well, and cultured overnight at $37{ }^{\circ} \mathrm{C}$ in air with $5 \% \mathrm{CO}_{2}$ and $95 \%$ humidity. The IPLLs were isolated from mice 22 weeks after immunization by standard protocols ${ }^{17}$. After overnight incubation, plates were washed with PBS $(\times 3)$ and PBS $+0.05 \%$ Tween $(\times 3)$ before addition of $100 \mu$ l of a 1:500 dilution of peroxidase-conjugated goat anti-mouse IgA (SBA) for $1 \mathrm{~h}$. Plates were developed with 3 amino-9-ethylcarbazole substrate reagent (Pierce). Red-brown-coloured spots were counted with the aid of a dissecting microscope (Zeiss). The lymphocyte isolation method consistently yielded $\sim 1-2 \times 10^{6}$ cells per mouse with $>98 \%$ viability.
Peyer's patches isolated from the gastrointestinal tract of i.n.immunized mice revealed lymphoid accumulations in follicles underlying the domed epithelium which were grossly larger than lymphoid aggregates seen in Peyer's patches of unimmunized or i.p.-immunized mice (data not shown). Lymphocytic infiltrates were also seen in the lamina propria and muscularis mucosa of i.n.-immunized mice, and large numbers of lymphocytes were seen extending into the villi as well.

Dense foci of lymphocytes were also observed in the nasopharyngeal-associated lymphoid tissue (NALT) of i.n.-immunized mice. Comparable lymphoid aggregates were absent in unimmunized mice or mice immunized i.p. (Fig. $3 e-g$ ). The appearance of organized lymphoid cell accumulations in NALT correlated with the appearance of a secretory immune response to the OspA antigen. The induction of organized lymphoid aggregates in NALT suggests a role for NALT in the generation of systemic and secretory responses against inhaled antigens.

At this point it is uncertain whether the appearance of lymphocytic foci in NALT and in distal tissues is due either to uptake of BCG by NALT microfold-cell equivalents followed by dissemination of $\mathrm{BCG}$ to distal tissues, or to stimulation of

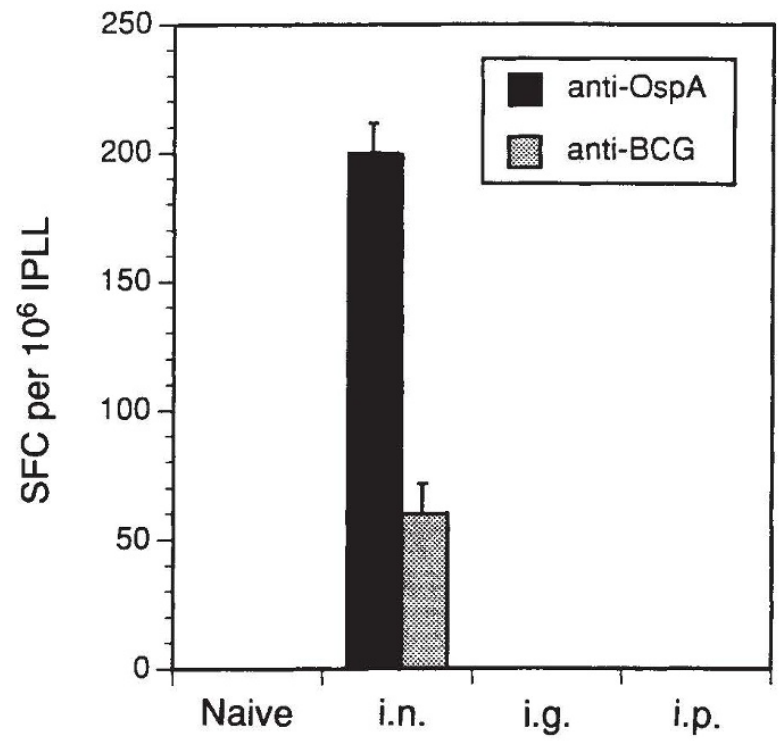

Route of immunization 

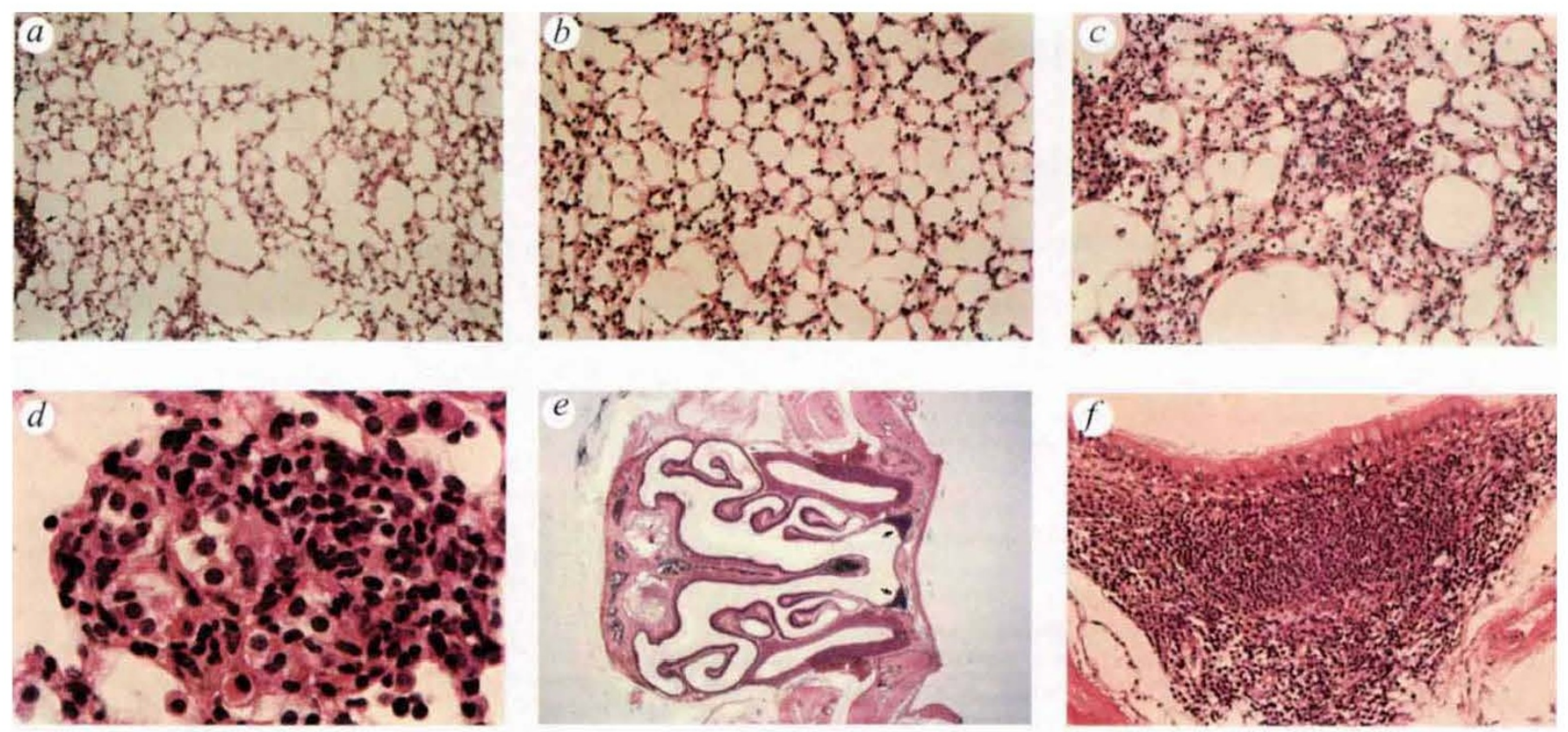

FIG. 3 Mouse lung and nasopharyngeal lymphoid tissue sections stained with haematoxylin and eosin. a, Section taken 6 weeks after i.n. immunization with $10^{8}$ CFU rBCG-OspA. Note regions of significant peribronchial lymphocytic infiltrates at the terminal bronchi (arrow) in otherwise normal-looking lungs. Magnification, $\times 76$. Similar histological changes were seen in mice immunized i.n. with non-recombinant BCG. $b$, Section taken 6 weeks after i.p. immunization with $10^{8} \mathrm{CFU}$ rBCG-OSPA. No regions of localized lymphocytic infiltrates were seen. Sections from non-immunized mice appeared to be the same as those taken from the i.p.immunized mice. Magnification, $\times 76$. Close-up views of $c$, peribronchial and periarterial lymphocytic infiltrates seen in the lungs of i.n.-immunized mice (magnification, $\times 190$ ), and $d$, one of the lymphoid aggregates (magnification, $\times 380$ ). Note the diverse population of lymphoid cells including monocytes, macrophages and lymphocytes within the aggregate. e, Cross-section through mouse nasal passages, at the level of the ecto- and endoturbinates ${ }^{25,26}$, revealing bipolar distribution of nasopharyngeal lymphoid tissue (NALT; arrows);

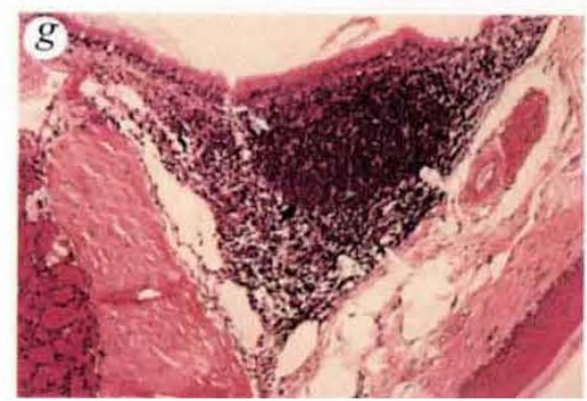
magnification, $\times 7.6$. $f$, Section of NALT from non-immunized mouse (magnification, $\times 190$ ). $g$, Section of NALT taken 6 weeks after i.n. immunization with $10^{8}$ CFU rBCG-OspA (magnification, $\times 95$ ). Note the dense accumulation of highly organized lymphoid tissue (arrow).

lymphocytes in the nasal mucosa followed by recirculation and homing to other mucosal tissues. Acid-fast and auromine-O staining of serial tissue sections from nasopharyngeal-, bronchial- and gut-associated lymphoid tissue as well as from spleen and liver tissue failed to reveal any BCG at these sites. However, quantitative culturing of lung and spleen tissue from i.n.-immunized mice has shown BCG persistence in lungs and spleen at least 9 weeks post-immunization. Converscly, although BCG was found in both spleens and lungs of i.p.-immunized mice at 1 day, and at 2 or 4 weeks post-immunization, rBCG-OspA was isolated only from spleens of i.p.-immunized mice at 9 weeks post-immunization. This finding suggests that persistence of BCG at proximal and distal sites of the mucosal immune system may be responsible in part for the sustained local immunity. Whether the NALT is also a primary site for stimulation of antigen-specific lymphocytes which then home to distal mucosal sites (for example, the gastrointestinal and genitourinary tracts) $)^{20.22}$, as well as to the spleen, is being investigated.

We conclude that intranasal delivery of $\mathrm{rBCG}-\mathrm{OspA}$ results in a potent, long-lasting systemic antibody responsc and a strong, pan-mucosal secretory IgA response to the vaccine. Although OspA was chosen as a model antigen to evaluate induction of immune responses following mucosal delivery of rBCG, we recognize that $B$. lurgdorferi does not infect through a mucosal surface. Nonetheless, our findings are significant in that they demonstrate that i.n. immunization with $\mathrm{rBCG}$ can elicit complete protection against systemic infection by the native organism cxpressing that same antigen, while inducing a sustained mucosal immune response. It remains to be determined whether mucosal immunization with $\mathrm{rBCG}$ expressing an antigen from a mucosal pathogen can protect against infection at a mucosal site.

Received 22 June; accepted 27 October 1994.

1. Jacobs, W. R. Jr, Tuckman, M. \& Bloom, B. R. Nature 327, 532-535 (1987).

2. Jacobs, W. R. Jr, Snapper, S. B., Lugosi, L. \& Bloom, B. R. Curr. Top. Microbiol. trmmun. 155, 153-160 (1990)

3. Stover, C. K. et at. Nature 351, 456-460 (1991)

4. Stover, C. K., Bansel, G. P.. Langermann, S. \& Hanson, M. S. in Recombinant Vectors in Vaccine Development (ed. Brown, F.) 153-160 (Karger, Basel, 1994).

5. Fujimura, Y. Gastroenterologica Jap. 21, 325-335 (1986).

6. Trier, J. S. Gastroenteroi. Clin. North Am. 20, 531-547 (1991).

7. Neutra, M. R. \& Kraenbuhl, J. P. Physiol. Rev. 2, 134-138 (1992).

8. Langermann, S. \& Amerongen, H. M. Curr. Opin. Gastroenterol. 8, $983-987$ (1993)

9. Bergstrom, S., Bundoc, V. \& Barbour, A. G. Molec. Microbiol. 3, 479-486 (1989)

10. Stover, C. K. et at. J. exp. Med. 178, 197-209 (1993).

11. Simon, M. M. et al. $J$. infect. Dis. 164, 123-132 (1991)

12. Fikrig, E., Barthold, S. W. Kantor, F. S. \& Flazvell, R. Science 280, 553-556 (1990).

13. Sadziene, A. Thompson, P. A. \& Barbour, A. G. J. infect. Dis. 167, 16.5-172 (1993)

14. Schwann, T. G. et at. J. clin. Microbial. 26, 893-895 (1988).

15. Abraham, E. \& Shah, S. J. immun. 149, 3719-3726 (1992).

16. Vajdy, M. \& Lycke. N. Y. Immunology 75, 488-492 (1992)

17. Vajdy, M. \& Lycke, N. Y. Immunology 75, $488-492$

17. O'Hagan, D. T. ot al. Vaccine 11, 149-154 (1993).
18. Quiding. M. et at. J. clin. Invest. 88, 143-148 (1991).

19. Haneberg. B. et al. infect. immun. 62, 15-23 (1994).

20. McDermott, M. R. \& Bienenstock, J. J. immun. 122, 1892-1898 (1979].

21. Fuhrman, J. A. \& Cebra, J. J. J. exp. Med. 153, 534-544 (1981).

22. Czerkinsky, C. et ai. Proc. natn. Acad. Sci. U.S.A. 84, 2449-2453 (1987).

23. Czerkinsky, C. et al. L. immun. Meth. 65, 109-121 (1983).

24. Sedgwick, J. D. \& Holt, P. G. J. immun. Meth. 87, 301-307 (1983).

25. Young, J. T. Fund apot. Toxicol. 1, 309-312 (1981).

26. Kuper, C. F. ot ai. immun. Today 13, 219-225 \{1992\}.

ACKNOWLEDGEMENTS. We thank C. Fox for assistance with histology: A. Barbour, J. Orenstein A. Anderson and M. Roy for discussion; H. Kiyono, J. McGnee and K. Fujihashi for advice on the ELISPOT assay: L. Erdile for OSpA; N. Patel for BCG vaccins preparations; and D. Leach tor assistance. 